\title{
CARACTERÍSTICAS DAS MÃES E DOS RECÉM-NASCIDOS EM MUNICÍPIOS-SEDE DE REGIONAL DE SAÚDE NO PARANÁ A PARTIR DOS DADOS DO SINASC
}

\author{
Emiliana Cristina Melo ${ }^{1}$, Thais Aidar de Freitas Mathias ${ }^{2}$
}

\begin{abstract}
RESUMO: Foram analisadas características dos nascidos vivos e mães residentes em municípios-sede de Regionais de Saúde no Paraná, em 2006, a partir do Sistema de Informações de Nascidos Vivos, segundo variáveis que podem representar desvantagem em saúde. Nos municípios-sede, em média, 19,4\% das mães eram adolescentes, 35,9\% tinham baixa escolaridade, 26,4\% tiveram número insuficiente de consultas de pré-natal e 55\% dos partos foram por cesariana. Existe heterogeneidade entre os municípios no Paraná, destacando-se Guarapuava, Paranaguá e Umuarama com elevados percentuais indicativos de risco à mãe e ao bebê. Estes resultados evidenciam municípios que necessitam de maiores recursos e políticas públicas específicas, descentralizadas e integralizadas, envolvendo gestores, profissionais de saúde e movimentos sociais organizados, no intuito de minimizar as desigualdades na saúde da população, em especial da população materno-infantil.
\end{abstract}

PALAVRAS-CHAVE: Nascimento vivo; Enfermagem em saúde comunitária; Sistemas de informação

\section{MOTHER'S AND NEWBORN CHARACTERISTICS IN MAIN MUNICIPALITIES OF REGIONAL HEALTH UNITS IN THE STAT OF PARANÁ FROM SINASC'S DATA FILES}

\begin{abstract}
SINASC'S data were the basis to develop the profiles of newborn and mothers resident in cities which have the Health Regional Headquarters; these profiles were analyzed by the light of variables that might indicate some health disadvantages. In these cities, on average, $19,4 \%$ of mothers were teenagers, $35,9 \%$ had poor education; $26,4 \%$ had not enough prenatal consultations and 55\% of the baby deliveries were performed by a C-section. Cities in Parana State are heterogeneous, with some highlights to Guarapuava, Paranaguá and Umuarama which have high indication of risks for the mother and the baby. These results show that some cities need more resources and specific, decentralized and integrated public policies, involving their managers, health professionals and organized social movements, in order to mitigate health inequalities among population, specially women and children.
\end{abstract}

KEYWORDS: Live births; Community health nursing care; Information systems.

\section{CARACTERÍSTICAS DE LAS MADRES Y DE LOS RECIÉN -NACIDOS EN MUNICIPIOS- SEDE DE REGIONAL DE SALUD EN EL PARANÁ A PARTIR DE LOS DATOS DEL SINASC}

RESUMEN: Fueron analizadas características de los nacidos vivos y madres residentes en municipios sede de Regionales de Salud en el Paraná, en 2006, a partir del Sistema de Informaciones de Nacidos Vivos, segundo variables que pueden representar desventaja en salud. En los municipios sede, en media, 19,4\% de las madres eran adolescentes, 35,9\% tenían baja escolaridad, 26,4\% tuvieron número insuficiente de consultas de pre-natal y 55\% de los partos fueron por cesárea. Existe heterogeneidad entre los municipios en el Paraná, destacando Guarapuava, Paranaguá y Umuarama con elevados porcentajes indicativos de riesgo a la madre y al bebé. Estos resultados evidencian municipios que necesitan de mayores recursos y políticas públicas específicas, descentralizadas e integradas, envolviendo gestores, profesionales de salud y movimientos sociales organizados, con el intuito de minimizar las desigualdades en la salud de la populación, en especial de la población materno-infantil.

PALABRAS CLAVE: Nacimiento vivo; Enfermería en salud comunitaria; Sistemas de información.

${ }^{1}$ Enfermeira. Mestre em Enfermagem. Professora Auxiliar da Universidade Estadual do Norte do Paraná-UENP.

${ }^{2}$ Enfermeira. Doutora em Saúde Pública. Professor Adjunto do Departamento de Enfermagem da Universidade Estadual de MaringáUEM. Coordenadora do Programa de Mestrado em Enfermagem da UEM.

Autor correspondente:

Emiliana Cristina Melo

Universidade Estadual do Norte do Paraná

Rodovia BR 369, km 54 - 86360-000 - Bandeirantes-PR, Brasil

Recebido: 09/07/09

E-mail:emiliana_melo@hotmail.com

Aprovado: 12/05/10 


\section{INTRODUÇÃO}

A sobrevivência dos recém-nascidos e as suas condições de vida são influenciadas por fatores que englobam desde as condições de realização do prénatal, parto e nascimento até as condições ambientais e sociais, genéticas e culturais ${ }^{(1)}$. Análises do comportamento de variáveis como a escolaridade e idade da mãe, peso do recém-nascido, tipo de parto e número de consultas de pré-natal podem indicar a situação de saúde da comunidade e, consequentemente, fornecer subsídios para melhorar a qualidade do atendimento nos serviços de saúde ${ }^{(1)}$, em especial do atendimento à gestação e ao parto. Umas das fontes de dados importantes para essas análises são as estatísticas vitais. São dados coletados rotineiramente nos serviços de saúde e vão compor os Sistemas de Informação do Sistema Único de Saúde (SIS-SUS), como por exemplo, os dados da gestação, da mãe, do parto e do recém-nascido (RN).

O Sistema de Informação sobre Nascidos Vivos (Sinasc) é um dos principais bancos de dados de saúde do SUS. Implantado pelo Ministério da Saúde, em 1990, com o objetivo de diminuir a subenumeração e melhorar a qualidade da informação sobre os nascimentos, o Sinasc utiliza como instrumento a Declaração de Nascidos Vivos (DN), documento padronizado preenchido para todos os RN nascidos no Brasil ${ }^{(2)}$.

A DN possui dados da mãe, como escolaridade e idade; do pré-natal, como o número de consultas; da gestação, como o número de semanas gestacionais; e do RN, como o Apgar e peso ao nascer. Todos estes são dados que, quando analisados, permitem conhecer aspectos socioeconômicos, de atendimento à saúde e também aspectos da vitalidade do $\mathrm{RN}^{(2)}$. O banco de dados do Sinasc possui grande potencial, pois reúne oficialmente informações de todos os nascimentos vivos e, por isso, deve ser constantemente explorado pelos profissionais e pelas equipes de saúde, tanto em comunidades, como em nível municipal. Os resultados dessas análises podem fornecer diagnósticos de saúde e identificar grupos populacionais em situação de risco.

Entretanto, sempre que as estatísticas do Sinasc são utilizadas existe o questionamento relativo à sua confiabilidade, seja na cobertura ou quantidade, seja no preenchimento adequado da DN. Quanto à qualidade do Sinasc no Paraná, para o período de 2000 a 2005, foi verificado que todas as variáveis tiveram percentuais de não-preenchimento abaixo de 3\%, exceto a variável “ocupação da mãe” que apresentou percentual de 3\% a 7\% de não-preenchimento(3) ${ }^{(3)}$ Já em 2006, foi observado, para o conjunto dos municípios-sede de Regionais de Saúde (RS), que para "idade da mãe", "tipo de parto", "peso ao nascer" e "semanas de gestação", os percentuais de dados ignorados e não preenchidos foram de $0,01 \%$ a $0,05 \%$, respectivamente. Para as variáveis "número de consultas de pré-natal” e "escolaridade da mãe", esse percentual ficou entre $0,24 \%$ e $0,23 \%$, respectivamente ${ }^{(4)}$, demonstrando a potencialidade do Sinasc como fonte de informação em saúde no Estado.

O Estado do Paraná está dividido em 22 RS com os respectivos municípios-sede concentrando maior quantidade e complexidade de serviços de saúde. Oficialmente, elas devem assessorar os municípios no âmbito de sua área, absorvendo ações e serviços que os outros municípios de sua abrangência não possuem, influenciando a gestão regional em todas as áreas da saúde ${ }^{(5)}$. Nesta ótica, o presente estudo teve por objetivo analisar características dos nascidos vivos e de suas mães, residentes nos 22 municípios-sede de RS do Paraná em 2006. Espera-se, com os resultados, conhecer aspectos socioeconômicos e da assistência, identificando possíveis diferenças entre os municípios para subsidiar o bom planejamento e avaliação do atendimento à saúde materno-infantil.

\section{METODOLOGIA}

Este é um estudo exploratório, de tipo ecológico, por comparar indicadores sociais e de saúde da população, por áreas geográficas. As informações foram provenientes do Sinasc, para todos os nascidos vivos de mães residentes nos 22 municípios-sede de RS do Paraná, em 2006, último ano com informações disponíveis ${ }^{(4)}$. A forma de agrupamento das variáveis obedeceu a disponibilidade das informações no Sinasc e foram construídos os seguintes indicadores que podem representar risco para a saúde da mãe e do RN: socioeconômicos - mãe adolescente ( $<20$ anos); mãe com baixa escolaridade (< oito anos de estudo) e raça/cor negra (preta e parda); assistenciais - prematuridade ( $<37$ semanas de gestação), número insuficiente de consultas de pré-natal $(<$ sete) e parto cesáreo; e resultantes - baixo peso ao nascer (< 2500 g) e baixa vitalidade do RN (Apgar no quinto minuto < oito). A análise foi feita por meio de proporções, agrupadas em intervalos e comparadas aos percentuais médios dos municípios-sede, do Estado 
do Paraná e do Brasil. O projeto de pesquisa foi aprovado pelo Comitê de Ética em Pesquisa com Seres Humanos da Universidade Estadual de Maringá, parecer n. 361/2008, e está de acordo com a Resolução n. 196/96 do Conselho Nacional de Saúde.

\section{RESULTADOS E DISCUSSÃO}

Os resultados deste estudo mostram heterogeneidade, evidenciando municípios que apresentaram proporções mais elevadas de indicadores de risco ao nascimento.

No Paraná, em 2006 nasceram 153.598 bebês de mães residentes nos Estado, dos quais 70.129 (45,6\%) nos 22 municípios-sede de RS.

Com relação às variáveis socioeconômicas, o percentual médio de mães adolescentes, com baixa escolaridade e de raça/cor negra, nos 22 municípiossede de RS, foi de $19,4 \%$, 36\% e 7,3\%, respectivamente. Merece destaque o Município de Telêmaco Borba com percentuais de mães adolescentes (27,1\%), com baixa escolaridade $(62,7 \%)$ e de raça/cor negra (35,8\%) mais elevados (Quadro 1). Segundo dados do Datasus ${ }^{(6)}$, a taxa de mortalidade infantil nesse município, para o ano de 2006, foi de 16,9 óbitos por mil nascidos vivos, superior à média dos municípiossede que foi de 12,7 óbitos por mil nascidos vivos, e maior que a média paranaense (14 óbitos por mil nascidos vivos). Tal constatação indica maior vulnerabilidade para as gestantes e $\mathrm{RN}$ residentes em Telêmaco Borba.

A gravidez na adolescência, a baixa escolaridade, o baixo peso ao nascer e o parto prematuro são fatores importantes que representam risco à saúde da mãe e do RN, tanto no nascimento como na infância. As consequências da gravidez na adolescência, por exemplo, vão desde maior possibilidade de baixo peso do bebê, e parto prematuro, até complicações sociais como a perda de oportunidade educacional e maior dificuldade em atividades produtivas que exigem maior qualificação, determinando a manutenção do ciclo de pobreza e perpetuação da gestação precoce nas descendentes ${ }^{(7-8)}$. Quanto à escolaridade, espera-se que com, no máximo 16 anos de idade, o tempo de estudo seja equivalente a 8 anos, período necessário para a conclusão do ensino fundamental ${ }^{(9)}$. Por outro lado, é importante atentar para a possibilidade das mães, ainda adolescentes, não terem completado a escolaridade desejável; mas este pode não ser o único fator determinante para a baixa escolaridade, já que pouco mais que $50 \%$ da população brasileira têm escolaridade ideal na idade prevista. Além disso, em municípios menores, a realidade ainda não condiz com as expectativas do Ministério da Educação, persistindo as desigualdades regionais ${ }^{(9)}$.

Quanto à variável raça/cor, 17 dos 22 municípios sede de RS apresentaram porcentagens inferiores a 10\% de negros (Quadro 1). Os Municípios de União da Vitória, Irati, Francisco Beltrão e Curitiba apresentaram percentuais inferiores a $1 \%$ de bebês negros, enquanto Telêmaco Borba (35,8\%), Jacarezinho (25,7\%) e Apucarana (24,3\%) apresentaram os percentuais mais elevados (Quadro 1).

De acordo com o Censo de 2000 o Brasil possuía uma população de 10 milhões de indivíduos negros (6,2\% da população total do País) e 65 milhões de pardos (38,4\%), totalizando 44,6\% de brasileiros negros e pardos, e para o Paraná esse percentual foi de pouco mais que $5 \%{ }^{(10)}$. Proporções mais baixas de população negra no Paraná podem ser justificadas pela predominante colonização espanhola e portuguesa. A partir do século XIX, além dos europeus, vieram paulistas, mineiros e nordestinos para o estado, podendo justificar esses percentuais mais baixos de negros e pardos quando comparados a outras regiões do Brasil ${ }^{(11)}$. É necessário lembrar que mesmo com mudanças de conceito e preconceitos raciais, no Brasil ainda são marcantes as diferenciações na qualidade de vida da população negra e, mesmo no Paraná, com percentuais abaixo da média nacional, o acúmulo das desigualdades sofridas pelos negros indica maior vulnerabilidade, em especial das crianças, que são mais propensas às iniquidades ${ }^{(12)}$.

A análise das variáveis assistenciais evidenciou que nos municípios estudados, em média, 7,4\% dos nascimentos ocorreram antes de 37 semanas de gestação, 26,4\% das mães realizaram menos de sete consultas de pré-natal e a forma preferencial para o parto foi a cesariana (55\%). Alguns municípios como Cornélio Procópio, Umuarama, Maringá e, em especial, Ivaiporã, estiveram entre os municípios com maior porcentagem de prematuridade e também de parto cesáreo (Quadro 2).

As taxas de parto cesáreo nos municípios-sede de RS ultrapassam, em muito, o percentual preconizado pelo Ministério da Saúde que corresponde a 15\% dos partos $^{(13)}$. Altas taxas de parto cesáreo podem implicar em maior tempo de permanência da mãe e do bebê no hospital, recuperação puerperal tardia, consumo de medicamentos e recursos tecnológicos para o parto e 
ainda podem representar risco para morbimortalidade materna e infantil ${ }^{(14)}$. As desvantagens para os recémnascidos de parto cesáreo incluem prematuridade, baixo peso e consequências como maior probabilidade de permanência em Unidades de Terapia Intensiva ${ }^{(8)}$.

Estudos demonstram a possibilidade dos partos Quadro 1 - Distribuição das variáveis socioeconômicas em municípios sede de Regionais de Saúde. Paraná, 2006.

\begin{tabular}{|c|c|c|}
\hline \multicolumn{3}{|c|}{ Mães adolescentes } \\
\hline Até $16,9 \%$ & 17 a $19 \%$ & $20 \%$ e mais \\
\hline 13,5 Maringá & 17,5 Paranvaí & 20,1 Apucarana \\
\hline 14,0 Cianorte & 17,7 Umuarama & 20,3 Cascavel \\
\hline 14,8 C.Procópio & 18,2 Toledo & 20,4 P.Grossa \\
\hline 15,7 Curitiba & 18,6 P.Branco & 20,7 F.Iguaçu \\
\hline 16,9 F.Beltrão & 18,7 Irati & 21,0 C.Mourão \\
\hline 16,9 Londrina & & 21,8 U.Vitória \\
\hline & & 22,4 Paranaguá \\
\hline & & 22,6 Jacarezinho \\
\hline & & 23,4 Ivaiporã \\
\hline & & 24,4 Guarapuava \\
\hline & & 27,1 T.Borba \\
\hline \multicolumn{3}{|c|}{ Média dos municípios-sede: $19,4 \%$} \\
\hline \multicolumn{3}{|c|}{ Média do Estado do Paraná: 20,7\%* } \\
\hline \multicolumn{3}{|c|}{ Média do Brasil: $21,5 \% *$} \\
\hline \multicolumn{3}{|c|}{ Mães com baixa escolaridade } \\
\hline Até $30 \%$ & 31 a $34 \%$ & $35 \%$ e mais \\
\hline 15,9 Maringá & 31,0 Cascavel & 35,4 Jacarezinho \\
\hline 21,0 Curitiba & 32,4 Umuarama & 36,7 P.Grossa \\
\hline 27,3 C.Mourão & 34,4 Apucarana & 37,0 F.Iguaçu \\
\hline 27,5 F.Beltrão & 34,6 U.Vitória & 37,3 C.Procópio \\
\hline 27,7 Londrina & & 37,3 Toledo \\
\hline 29,1 Paranavaí & & 38,9 P.Branco \\
\hline & & 39,2 Irati \\
\hline & & 43,8 Paranaguá \\
\hline & & 44,8 Ivaiporã \\
\hline & & 47,4 Guarapuava \\
\hline & & 49,4 Cianorte \\
\hline & & 62,7 T.Borba \\
\hline \multicolumn{3}{|c|}{ Média dos municípios-sede: $35,9 \%$} \\
\hline \multicolumn{3}{|c|}{ Média do Estado do Paraná: 38,3\%* } \\
\hline \multicolumn{3}{|c|}{ Média do Brasil: 43,9\%* } \\
\hline
\end{tabular}

Continua

\begin{tabular}{|c|c|c|}
\hline \multicolumn{3}{|c|}{ Raça/cor negra } \\
\hline Até 3\% & 3,1 a $6,9 \%$ & $7 \%$ e mais \\
\hline 0,0 U.Vitória & 3,1 F.Iguaçu & 7,2 Umuarama \\
0,1 Irati & 4,4 Toledo & 9,3 Paranavaí \\
0,2 F.Beltrão & 5,8 Ivaiporã & 11,3 C.Procópio \\
0,4 Curitiba & 6,8 Maringá & 11,9 Londrina \\
1,0 P.Grossa & & 24,3 Apucarana \\
1,6 C.Mourão & & 25,7 Jacarezinho \\
1,7 P.Branco & & 35,8 T.Borba \\
1,9 Paranaguá & \\
2,3 Cianorte & \\
2,3 Guarapuava & \\
2,7 Cascavel & \\
\hline Média dos municípios-sede: 7,3\% \\
Média do Estado do Paraná: $5,8 \% *$ \\
\hline Média do Brasil: 46,6\%*
\end{tabular}

*Fonte: Ministério da Saúde (BR). DATASUS: informações de saúde: estatísticas vitais: nascidos vivos. Paraná, 2006.

cesáreos ocorrerem por conveniência e sua associação com melhores condições socioeconômicas das mães que, por sua vez, têm maiores chances da opção ou indicação da cesariana, facilitadas pelo pagamento do parto e negociação com o obstetra ${ }^{(14-15)}$. Assim, a taxa de cesarianas deve ser analisada por vários ângulos, tanto como indicador de acesso aos serviços de saúde, quanto como possível fator condicionante ou coadjuvante no aumento do parto prematuro e do baixo peso ao nascer.

No entanto, a prematuridade e suas consequências não podem ser associadas somente ao parto cesáreo. Estudo realizado em Pelotas, no Rio Grande do Sul, aponta como possível causa da prematuridade as infecções maternas que desencadeiam o trabalho prematuro de parto, como os casos de infecções do trato urinário(15)

Quanto às consultas de pré-natal, merecem destaque os Municípios de Guarapuava, Paranaguá e Foz do Iguaçu onde quase metade das gestantes (mais de $45 \%$ ) realizaram menos de sete consultas. Por outro lado os Municípios de Cianorte com 6\% e Apucarana com $9,3 \%$ foram os que mais ofereceram consultas de pré-natal às suas gestantes (Quadro 2). Esses resultados mostram as diferenças existentes entre os municípios-sede de RS no Estado do Paraná, que podem refletir diferenciais no acesso aos serviços pela população na quantidade de clínicas e unidades básicas 
de saúde existentes nesses municípios.

No Brasil, em 2006, aproximadamente $44 \%$ das gestantes realizaram menos de sete consultas durante Quadro 2 - Distribuição das variáveis assistenciais em municípios sede de Regionais de Saúde. Paraná, 2006.

\begin{tabular}{|c|c|}
\hline \multicolumn{2}{|c|}{ Prematuridade } \\
\hline Até $6,9 \%$ & $7 \%$ e mais \\
\hline 2,9 Jacarezinho & 7,3 Apucarana \\
\hline 4,4 Irati & 7,5 P. Branco \\
\hline 4,8 Cianorte & 7,6 C. Mourão \\
\hline 5,4 Paranaguá & 8,0 Toledo \\
\hline 5,5 T. Borba & 8,3 Maringá \\
\hline 5,6 Guarapuava & 8,3 Cascavel \\
\hline 5,8 Paranavaí & 8,6 C. Procópio \\
\hline 5,9 Ponta Grossa & 9,2 Londrina \\
\hline 6,1 Foz do Iguaçu & 9,9 Umuarama \\
\hline 6,4 F. Beltrão & 21,4 Ivaiporã \\
\hline 6,7 Curitiba & \\
\hline 6,9 União da Vitória & \\
\hline \multicolumn{2}{|c|}{ Média dos municípios-sede: $7,4 \%$} \\
\hline \multicolumn{2}{|c|}{ Média do Estado do Paraná: 6,6\%* } \\
\hline \multicolumn{2}{|l|}{ Média do Brasil: $6,6 \% *$} \\
\hline \multicolumn{2}{|c|}{ Número insuficiente de consultas de pré-natal } \\
\hline Até $29 \%$ & $30 \%$ e mais \\
\hline 6,0 Cianorte & 30,6 Jacarezinho \\
\hline 9,3 Apucarana & 31,3 T. Borba \\
\hline 12,3 F. Beltrão & 34,7 Ivaiporã \\
\hline 12,7 Ponta Grossa & 35,1 Cascavel \\
\hline 13,2 Londrina & 35,3 Irati \\
\hline 13,4 Curitiba & 38,7 Toledo \\
\hline 16,2 C. Procópio & 46,2 F. Iguaçu \\
\hline 17,2 Maringá & 47,7 Paranaguá \\
\hline 20,5 Umuarama & 51,3 Guarapuava \\
\hline 22,8 C. Mourão & \\
\hline 26,9 P. Branco & \\
\hline 28,7 U. Vitória & \\
\hline 28,8 Paranavaí & \\
\hline \multicolumn{2}{|c|}{ Média dos municípios-sede: $26,4 \%$} \\
\hline \multicolumn{2}{|c|}{ Média do Estado do Paraná: 24,9\%* } \\
\hline Média do Brasil: 43,9\%* & \\
\hline
\end{tabular}

Continua

\begin{tabular}{|c|c|}
\hline \multicolumn{2}{|c|}{ Parto cesáreo } \\
\hline Até 50\% & $51 \%$ e mais \\
\hline 40,9 Irati & 51,0 Cianorte \\
41,2 Guarapuava & 53,2 P. Branco \\
41,4 P. Grossa & 53,6 Paranaguá \\
45,3 T. Borba & 55,1 Jacarezinho \\
46,4 U. Vitória & 55,1 Londrina \\
47,5 F. Iguaçu & 56,5 Curitiba \\
47,6 F. Beltrão & 57,6 Paranavaí \\
50,8 Cascavel & 59,7 C. Mourão \\
& 60,4 Toledo \\
& 65,8 Apucarana \\
& 66,7 Ivaiporã \\
\hline
\end{tabular}

*Fonte: Ministério da Saúde (BR). DATASUS: informações de saúde: estatísticas vitais: nascidos vivos. Paraná, 2006.

a gestação, as regiões Sul e Sudeste com 29\% e 31\%, respectivamente, enquanto as regiões Norte e Nordeste apresentaram entre $59 \%$ e $69 \%{ }^{(5)}$. A maior parte dos municípios-sede de RS no Paraná teve percentuais insuficientes de consultas de pré-natal, inferiores à média nacional e das regiões Sul e Sudeste. No entanto, alguns desses municípios ainda necessitam maiores investimentos na atenção à saúde da mulher e da criança para cumprirem o mínimo de consultas preconizado, considerando que o acompanhamento pré-natal satisfatório diminui as complicações na gestação, parto e puerpério, e a morbi-mortalidade infantil ${ }^{(7,15)}$

O Programa de Humanização do Pré-Natal, Parto e do Nascimento (PHPN) ${ }^{(13)}$ recomenda, no mínimo, seis consultas, com início do pré-natal no primeiro trimestre da gestação. Por outro lado, estudo revela que, na opinião de enfermeiros da Estratégia Saúde da Família, o número mínimo de seis consultas ainda não é suficiente para o desenvolvimento de todas as ações preconizadas durante o pré-natal ${ }^{(16)}$, demonstrando não só a necessidade de alcançar a cobertura de pré-natal preconizada, mas também de superá-la. É importante observar que alguns municípios com altos 
percentuais de mães com menos de sete consultas de pré-natal foram o mesmos com percentuais acima de $45 \%$ de mães com menos de oito anos de escolaridade (Guarapuava, 47,4\%; Cianorte, 49,4\%; e Telêmaco Borba, $62,7 \%$ ) e mais de $23 \%$ de mães adolescentes (Telêmaco Borba, 27,1\%; e Guarapuava, 24,4\%).

Mães adolescentes e com baixa escolaridade constituem um grupo vulnerável, prioritário para os serviços de saúde. Entende-se que a baixa escolaridade significa menor acesso à informação, determinando também menores condições de acesso aos serviços de saúde; as mães adolescentes com baixa escolaridade, por pertencerem, em geral, a classes sociais menos favorecidas, podem ter atendimento deficiente durante a gestação $0^{(15)}$. É importante ressaltar que a idade, escolaridade e classe social não são os únicos fatores já que o acesso à saúde é determinado por um conjunto de fatores sociais, econômicos e de políticas públicas adequadas que garantam o direito do indivíduo não só à assistência, mas à saúde e à qualidade de vida. Assim, a combinação encontrada nesses municípios, de altos percentuais de mães com baixa escolaridade, de mães adolescentes e de gestantes com número insuficiente de consultas de pré-natal, chama atenção para a necessidade de pronta intervenção dos gestores, pois provavelmente os indicadores de morbimortalidade podem também estar desfavoráveis.

Quanto ao alto percentual de menos que sete consultas de pré-natal nos Municípios de Paranaguá e Foz do Iguaçu, é importante tecer algumas considerações. Paranaguá, município portuário, apresentou nos últimos 40 anos um quadro de crescimento populacional acelerado e desigual no espaço urbano, criando uma diversificação que compõe um panorama de heterogeneidades espaciais e desigualdades sociais ${ }^{(17)}$.

Estudo realizado em 2000 mostra que os dados de mortalidade e morbidade de Paranaguá revelam situação desfavorável comparada ao Estado do Paraná, persistindo problemas como altas taxas de mortalidade infantil $^{(18)}$. Na assistência à saúde, embora pareça ter ocorrido um avanço do acesso da população aos serviços básicos, ações importantes como a assistência adequada à gestação e ao parto ainda parecem não ser extensivas a toda a população $0^{(17)}$.

Foz do Iguaçu, um dos municípios-sede de RS com maior percentual de mães com baixa cobertura de pré-natal (46,2\%), possui características específicas por fazer fronteira com o Paraguai e Argentina. A busca de atendimento pelo SUS por populações residentes em regiões de fronteira - e também por brasileiros residentes no exterior - é frequente para todos os tipos de serviços de saúde, dentre eles a atenção pré-natal. É comum estrangeiros e brasileiros que moram em países vizinhos utilizarem comprovantes de residência de parentes e amigos que moram nos municípios de fronteira como Foz do Iguaçu ${ }^{(18)}$. Neste caso, o acesso torna-se mais difícil pela distância relativa, necessidade de transporte para o deslocamento e maior disponibilidade de tempo por parte da gestante, que pode comparecer apenas ao mínimo de consultas para garantir o parto pelo SUS.

Esses resultados mostram desigualdades importantes entre os municípios, que devem ser evidenciadas e levadas em consideração pelos gestores na previsão e distribuição de recursos e serviços, bem como na qualificação de profissionais de saúde. Enquanto o percentual de mães adolescentes foi de 13,5\% para Maringá, para Telêmaco Borba foi de 27,1\%; quanto a mães com baixa escolaridade, foi de 15,9\% para Maringá e de 62,7\% para Telêmaco Borba; em União da Vitoria não foi registrado no Sinasc nenhum RN de raça/cor negra, mas houve 35,8\% em Telêmaco Borba; houve 6\% de gestantes com menos de sete consultas de pré-natal em Cianorte e 51,3\% para o Município de Guarapuava.

Ao analisar as variáveis resultantes, observase que o percentual de baixo peso esteve entre $4,3 \%$ (Ivaiporã) e 10,4\% (Apucarana), com média de 8,1\%. Em países desenvolvidos esse percentual não ultrapassa os $6 \%{ }^{(12)}$. No Brasil, em 2006 a proporção de baixo peso ao nascer foi de $8,2 \%{ }^{(4)}$, a mesma encontrada para o conjunto dos municípios-sede de RS estudados (Quadro 3).

O baixo peso ao nascer expõe o recém-nascido a riscos de morbimortalidade e constitui um indicador de saúde que, além de permitir comparações, fundamenta ações preventivas, já que as situações que desencadeiam um parto prematuro podem ser detectadas durante a gestação ${ }^{(15)}$. Municípios que apresentaram valores entre intermediário e alto (maior que 8,1\%) para baixo peso, também são os mesmos com valores entre intermediário e alto de prematuridade (maior que 8,0\%) e de parto cesáreo (mais de 50\%) como Maringá, Umuarama, Toledo e Londrina, podendo indicar possível associação entre prematuridade, baixo peso e parto cesáreo ${ }^{(19)}$. Foi observada média de 2,2\% de baixa vitalidade do RN, com o maior percentual para Ponta Grossa (5,9\%) seguido por Guarapuava (3,4\%), Umuarama (3,3\%) e Campo Mourão (2,6\%). Os Municípios de Cornélio 
Procópio, Cianorte, Irati e Maringá tiveram percentuais abaixo de 1,5\% de nascidos vivos com baixa vitalidade (Quadro 3). O valor do Apgar no quinto minuto revela o estado de oxigenação e vitalidade do recém-nascido no período anterior e durante o parto, sendo que valores até sete indicam anóxia neonatal e entre 8 e 10, ausência desta. O Apgar insatisfatório, além de indicar o aumento na demanda de recursos tecnológicos e Quadro 3 - Distribuição das variáveis resultantes em municípios sede de Regionais de Saúde. Paraná, 2006.

\begin{tabular}{|c|c|c|}
\hline \multicolumn{3}{|c|}{ Baixo peso ao nascer } \\
\hline Até $8,1 \%$ & 8,2 a $9 \%$ & Mais de $9 \%$ \\
\hline 4,3 Ivaiporã & 8,2 Maringá & 9,1 Guarapuava \\
\hline 6,4 Cianorte & 8,2 T.Borba & 9,7 Toledo \\
\hline 6,9 C.Procópio & 8,2 U.Vitória & 10,4 Apucarana \\
\hline 7,5 F.Iguaçu & 8,4 Cascavel & \\
\hline 7,5 Jacarezinho & 8,5 Londrina & \\
\hline 7,8 Paranaguá & 8,5 C.Mourão & \\
\hline 7,9 Paranavaí & 8,6 P.Branco & \\
\hline 8,1 Irati & 8,6 Umuarama & \\
\hline 8,1 F. Beltrão & 8,9 Curitiba & \\
\hline & 8,9 P.Grossa & \\
\hline \multicolumn{3}{|c|}{ Média dos municípios-sede: $8,1 \%$} \\
\hline \multicolumn{3}{|c|}{ Média do Estado do Paraná: 8,2\%* } \\
\hline \multicolumn{3}{|c|}{ Média do Brasil: $8,2 \% *$} \\
\hline \multicolumn{3}{|c|}{ Baixa vitalidade do $\mathrm{RN}$} \\
\hline Até $2,2 \%$ & 2,3 a $2,4 \%$ & $2,5 \%$ e mais \\
\hline 1,2 C.Procópio & 2,3 Paranaguá & 2,5 Ivaiporã \\
\hline 1,2 Cianorte & 2,3 U.Vitória & 2,6 C.Mourão \\
\hline 1,3 Irati & 2,3 Cascavel & 3,3 Umuarama \\
\hline 1,4 Maringá & 2,3 T.Borba & 3,4 Guarapuava \\
\hline 1,5 F.Iguaçu & 2,4 Curitiba & 5,9 P.Grossa \\
\hline 1,6 Jacarezinho & 2,4 F.Beltrão & \\
\hline 1,6 Paranavaí & & \\
\hline 1,7 Toledo & & \\
\hline 1,9 Apucarana & & \\
\hline 1,9 Londrina & & \\
\hline 2,2 P.Branco & & \\
\hline \multicolumn{3}{|c|}{ Média dos municípios-sede: $2,2 \%$} \\
\hline \multicolumn{3}{|c|}{ Média do Estado do Paraná: 2,5\%* } \\
\hline & & \\
\hline
\end{tabular}

*Fonte: Ministério da Saúde (BR). DATASUS: informações de saúde: estatísticas vitais: nascidos vivos. Paraná, 2006. humanos, ainda expõe o $\mathrm{RN}$ a maior risco de morbimortalidade neonatal $^{(15)}$, situação que pode ser minimizada com um pré-natal adequado, permitindo a detecção e tratamento de possíveis situações de risco e do atendimento especializado na hora do nascimento.

Neste estudo, percebeu-se a existência de possível associação do baixo peso ao nascer com as variáveis assistenciais e socioeconômicas, evidenciada pelos indicadores de Guarapuava, município que se destaca com elevado percentual de mães adolescentes (24,4\%), baixa escolaridade (47,4\%), com insuficientes consultas de pré-natal (51,3\%), Apgar menor que 8 no quinto minuto $(3,4 \%)$ e baixo peso ao nascer $(9,1 \%)$. No entanto, os resultados desfavoráveis apresentados pelo Município de Guarapuava merecem estudo mais detalhado, abrangendo aspectos específicos da saúde materno-infantil, já que segundo relatório do Instituto Paranaense de Desenvolvimento Econômico e Social, que descreve a tipologia dos municípios paranaenses, Guarapuava é considerado um município com médioalto grau de desenvolvimento socioeconômico e com excelente estrutura básica de atendimento à população ${ }^{(20)}$.

\section{CONSIDERAÇÕES FINAIS}

A análise de variáveis disponíveis nos bancos de dados do SUS, em geral, e do Sinasc, em particular, devem ser utilizadas e avaliadas, pois com esses dados é possível conhecer e monitorar constantemente a situação de saúde da população, identificar diferenças entre os municípios, mostrando resultados e reflexos da atenção à saúde prestada e oferecida em cada localidade, além de possíveis desigualdades na qualidade de vida, educação e assistência à saúde materno-infantil.

Neste estudo, os dados expressaram que apesar do Paraná apresentar bons indicadores de saúde quando comparado a outras regiões do Brasil, ainda persistem desigualdades entre os municípios mais importantes do estado. Esses resultados fornecem elementos que subsidiam a deteç̧ão de situações específicas e orientam o planejamento de ações para intervenção de situações de risco. Ações descentralizadas e integralizadas devem ser prioridade, abrangendo também setores privados e conveniados ao SUS. Ações conjuntas que envolvam os gestores, os profissionais de saúde e a população devem considerar as reais necessidades de cada município.

Os gestores regionais e municipais devem conhecer essa realidade, assim como as equipes de Saúde da Família e os enfermeiros devem estar atentos 
aos indicadores de saúde, proporcionando atendimento de qualidade, observando e intervindo sobre os indicadores de vulnerabilidade.

O enfermeiro é membro presente e indispensável na equipe de saúde e tem papel fundamental no cuidado à saúde da mulher e da criança, e deve reconhecer as características da população por ele atendida. Reconhecer e compreender os fatores sociais explorados neste estudo, como a baixa escolaridade, número de mães adolescentes e raça/cor negra, é imprescindível para qualificar e humanizar o atendimento e a intervenção da equipe de saúde e do enfermeiro. Para que a intervenção de enfermagem seja efetiva é necessária à compreensão de fatores sociais, econômicos e assistenciais, os quais são determinantes nos resultados da gestação, tanto para mãe quanto para o RN.

Além disso, conhecer as características da população materno-infantil em seu município ou região de atuação é um dos principais requisitos para que o profissional possa qualificar e facilitar a atenção prénatal, o que contribui para a eliminação dos riscos e o alcance de uma assistência de saúde com equidade. A análise das variáveis por município-sede de RS contribui para esse conhecimento, bem como das diferenças entre os municípios relacionadas a essas variáveis.

\section{REFERÊNCIAS}

1. Romero DE, Cunha CB. Avaliação da qualidade das variáveis epidemiológicas e demográficas do Sistema de Informações sobre Nascidos Vivos, 2002. Cad Saúde Pública. 2007;23(3):701-14.

2. Mello Jorge MHP, Gotlieb SLD, Soboll MLMS, Baldijão MFA, Latorre MRDO. O sistema de informação sobre nascidos vivos-SINASC. São Paulo: Centro Brasileiro para Classificação de Doenças. Núcleo de Estudos em População e Saúde. Universidade de São Paulo; 1992.

3. Silva GF. Qualidade do sistema de informações sobre nascidos vivos no estado do Paraná, 2000 a 2005 [dissertação]. Maringá (PR): Universidade Estadual de Maringá; 2008.

4. Ministério da Saúde (BR). Datasus: informações de saúde: estatísticas vitais: nascidos vivos. Paraná. [Internet] 2006 [acesso em 01 mar 2009]. Disponível: http://tabnet.datasus.gov.br/cgi/deftohtm.exe?sinasc/ cnv/nvpr.def

5. Secretaria de Estado da Saúde do Paraná (PR). Plano estadual de saúde. [Internet] 1997 [acesso em 17 set 2009]. Disponível: http://www.saude.pr.gov.br/RS/index.html/

6. Ministério da Saúde (BR). Datasus: informações de saúde: estatísticas vitais: Óbitos infantis. Paraná. [Internet] 2006 [acesso em 01 mar 2009]. Disponível: http://tabnet.datasus.gov.br/cgi/deftohtm.exe?sim/cnv/ nvpr.def

7. Gama SGN, Szwarcwald CL, Leal M. Experiência de gravidez na adolescência, fatores associados e resultados perinatais entre puérperas de baixa renda. Cad Saúde Pública. 2002;18(1):153-61.

8. Uchimura TT, Pelissari DM, Soares DFPP, Uchimura N, Santana RG, Morais CMS. Fatores de risco para o baixo peso ao nascer segundo as variáveis da mãe e do recémnascido, em Maringá - PR, no período de 1996 a 2002. Cienc Cuid Saúde. 2007;6(1):51-8.

9. Sampaio CEM, Nespoli V. Índice de adequação idadeanos de escolaridade. Rev Bras Est Pedag. 2004;85(209/ 210/211):137-42.

10. Instituto Brasileiro de Geografia e Estatística - IBGE. Censo demográfico 2000: características gerais da população: resultados da amostra. Rio de Janeiro; 2000.p.1-178. [acesso em 2008 Out 15]. Disponível: http:/ /www.ibge.gov.br/home/estatistica/populacao/censo20 00/populacao/censo2000_populacao.pdf

11. Wachowicz RC. História do Paraná. 9a ed. Curitiba: Imprensa Oficial do Paraná; 2001.

12. Fundo das Nações Unidas para a Infância - UNICEF. Situação mundial da infância 2008. Brasília (DF); 2008. [acesso em 2008 Jun 19] 20. Disponível: http://www.uni cef.org/brazil/pt/sowc2008_br.pdf

13. Ministério da Saúde (BR). Programa de humanização do pré-natal e nascimento. Secretaria de Políticas de Saúde. Departamento de Atenção Básica. Brasília: Ministério da Saúde;2000.

14. Souza Júnior JC, Kunkel N, Gomes MA, Freitas PF. Equidade inversa e desigualdades no acesso à tecnologia no parto em Santa Catarina, Brasil, 2000 a 2004. Rev Bras Saúde Matern Infant. 2007;7(4):397-403.

15. Coimbra LC, Silva AAM, Mochel EG, Alves MTSSB, Ribeiro VS, Aragão VM, et al. Fatores associados à inadequação do uso da assistência pré-natal. Cad Saúde Pública. 2003;37(4):456-62.

16. Benigna MJC, Nascimento WG, Maerins JL. Pré-natal no Programa Saúde da Família (PSF): com a palavra, os

Cogitare Enferm. 2010 Abr/Jun; 15(2):293-301 
enfermeiros. Cogitare Enferm. 2004;9(2):23-31.

17. Gerhardt TE. Situações de vida, pobreza e saúde: estratégias alimentares e práticas sociais no meio urbano. Ciênc Saúde Col. [Internet] 2003 [acesso em 13 nov 2009] 8(3):713-26. Disponível: http://www.scielosp.org/pdf/csc/ v8n3/17452.pdf.

18. Giovanella L, Guimarães L, Nogueira VMR, Lobato LVC, Damacena GN. Saúde nas fronteiras: acesso e demandas de estrangeiros e brasileiros não residentes ao SUS nas cidades de fronteira com países do MERCOSUL na perspectiva dos secretários municipais de saúde. Cad Saúde Pública. [Internet] 2007;23(Supl 2) [acesso em 18 nov 2009]. Disponível: http://www.scielo.br/pdf/\%0D/ csp/v23s2/13.pdf.

19. Souza Júnior JC, Kunkel JC, Gomes N, Freitas MA, Fontoura PF. Equidade inversa e desigualdades no acesso à tecnologia no parto em Santa Catarina, Brasil, 2000 a 2004. Rev Bras Saúde Matern Infant. 2007;7(4):397-403.

20. Instituto Paranaense de Desenvolvimento Econômico e Social - IPARDES. Tipologia dos municípios paranaenses segundo indicadores socioeconômicos e demográficos. Curitiba;2003. [acesso em 20 mais 2009]. Disponível: http://www.ipardes.gov.br/biblioteca/docs/ tipologia.pdf. 